\title{
Lattice Boltzmann model for simulating flows with multiple phases and components
}

\author{
Xiaowen Shan and Hudong Chen \\ Department of Physics and Astronomy, Dartmouth College, Hanover, New Hamphshire 03755
}

(Received 5 November 1992)

\begin{abstract}
A lattice Boltzmann model is developed which has the ability to simulate flows containing multiple phases and components. Each of the components can be immiscible with the others and can have different mass values. The equilibrium state of each component can have a nonideal gas equation of state at a prescribed temperature exhibiting thermodynamic phase transitions. The scheme incorporated in this model is the introduction of an interparticle potential. The dynamical rules in this model are local so it is highly efficient to compute on massively parallel computers. This model has many applications in large-scale numerical simulations of various types of fluid flows.
\end{abstract}

PACS number(s): 47.55.Kf, 02.70.+d, 05.70.Fl

There are many difficulties in numerically simulating multiphase fluids or immiscible fluids with conventional methods. These flows include, for example, a flow of a water and vapor mixture or an oil and water mixture. Not only different phases may merge, separate or break, they may also change from one phase to another during a first-order phase transition process.

It is known that fluid phenomena seen in nature are the statistical behaviors of their associated microsystems. The thermodynamic properties and the boundary conditions for a fluid system are, therefore, natural consequences of the dynamics in these physical microsystems. To compute various fluid flows, it is, however, impractical to simply follow the evolutions of these realistic physical microsystems because of far too many degrees of freedom not essential to their fluid behaviors. Fortunately, due to the nonunique correspondence between a fluid system and a microsystem, artificial microsystems may be constructed which are simple enough to be simulated on a computer, but yet contain all the required physics of a realistic fluid system. Based on this idea, a computational approach called lattice Boltzmann (LB) method has recently been developed [1-4]. The LB method is derived from the lattice gas automata (LGA) method [5-7] and inherited from the LGA some of its major advantages over the conventional computational methods. One important improvement in the LB method is that it can fully recover the Navier-Stokes equations at the macroscopic scale $[3,4]$. It is parallel in nature due to the fact that all the information transfer is local in time and space, so that it is most suitable for the massively parallel computers. In addition, since this method deals with the fluid properties at the microkinetic level, it is capable of handling complex boundary conditions and various thermodynamic properties of a fluid system, such as multiphase flows, in a relatively straightforward way.

Since Rothman and Keller [8] introduced the first LGA model for simulating immiscible fluids, there have been LGA and LB models constructed for simulating immiscible fluids [8-12]. However, there are problems in these models in dealing with components with different molec- ular mass values. More importantly, these models cannot be successfully extended to flows having thermodynamic phase transitions because of their non-Galilean invariant nature and unphysical interfacial properties. In this paper, we show how to overcome both of these problems and present an alternative LB model which has the ability to simulate flows with immiscible components as well as multiple phases.

In this LB model, the following lattice Boltzmann equation is solved for a fluid with $S$ total components on a two-dimensional (2D) hexagonal lattice or on a 4D face-centered hypercubic lattice $[6,7]$ :

$$
\begin{aligned}
& n_{a}^{\sigma}\left(\mathbf{x}+\hat{\mathbf{e}}_{a}, t+1\right)-n_{a}^{\sigma}(\mathbf{x}, t)=\Omega_{a}^{\sigma}(\mathbf{x}, t) \\
& \sigma=1, \ldots, S, \quad a=0, \ldots, b,
\end{aligned}
$$

where $n_{a}^{\sigma}(\mathbf{x}, t)$ is the single-particle distribution function for the $\sigma$ th component and $\Omega_{a}^{\sigma}(\mathbf{x}, t)$ is the collision term. The set of vectors $\left\{\hat{\mathbf{e}}_{a} ; a=0, \ldots, b\right\}$ are the possible velocities a particle can have in order to move from a lattice site to one of the $b$ numbers of the nearest-neighboring sites at each time step. $\left|\hat{\mathbf{e}}_{0}\right|=0$ is associated with the rest ("stopped") particles, and $\left|\hat{\mathbf{e}}_{a}\right|=c(a=1, \ldots, b)$, where $c$ is the lattice constant. For simplicity, we adopt a "single relaxation time" form for the collision term [3, $4,13,14]$,

$$
\Omega_{a}^{\sigma}(\mathbf{x}, t)=-\frac{1}{\tau_{\sigma}}\left[n_{a}^{\sigma}(\mathbf{x}, t)-n_{a}^{\sigma(\mathrm{eq})}(\mathbf{x}, t)\right]
$$

where $\tau_{\sigma}$ is the mean collision time for the $\sigma$ th component and determines its fluid viscosity $[13,4] . n_{a}^{\sigma(e q)}$ is the equilibrium distribution with a given functional form at site $\mathbf{x}$ and time $t$ [14]. An $\mathrm{H}$ theorem can be proved for this system for arbitrarily given form of $n_{a}^{\sigma(\mathrm{eq})}$, as long as this equilibrium distribution function is positive [15]. For the purpose of recovering correct fluid equations, the following form for $n_{a}^{\sigma(e q)}$ is adopted $[3,4]$, 


$$
\begin{aligned}
& n_{a}^{\sigma(\mathbf{e q})}=n^{\sigma}\left[\frac{1-d_{0}^{\sigma}}{b}+\frac{D}{c^{2} b} \hat{\mathbf{e}}_{a}: \mathbf{u}^{\sigma}+\frac{D(D+2)}{2 c^{4} b} \hat{\mathbf{e}}_{a} \hat{\mathbf{e}}_{a}: \mathbf{u}^{\sigma} \mathbf{u}^{\sigma}-\frac{D}{2 c^{2} b} \mathbf{u}^{\sigma 2}\right], a=1, \ldots, b \\
& n_{0}^{\sigma(\mathbf{e q})}=n^{\sigma}\left[d_{0}^{\sigma}-\frac{1}{c^{2}} \mathbf{u}^{\sigma 2}\right]
\end{aligned}
$$

where $n^{\sigma}=\sum_{a} n_{a}^{\sigma}$ and $\mathbf{u}^{\sigma}$ are respectively the number density and the averaged particle velocity for component $\sigma$ at $(\mathbf{x}, t)$ after collision. $d_{0}^{\sigma}(<1)$ is a constant and determines the compressibility of each component of the fluid [16]. For sufficiently small $\mathbf{u}^{\sigma}$, the above form for the equilibrium distribution function will be positive. It has been analytically shown, with the above choice [3, 4], that at the fluid scale, each of the components obeys the isothermal Navier-Stokes equations with an ideal gas equation of state, if

$$
\mathbf{u}^{\sigma}=\mathbf{u}=\frac{1}{\rho} \sum_{\sigma} m^{\sigma} \sum_{a} \hat{\mathbf{e}}_{a} n_{a}^{\sigma},
$$

with $\rho\left(=\sum_{\sigma} m^{\sigma} \sum_{a} n_{a}^{\sigma}\right)$ and $\rho$ u being, respectively, the total mass density and net momentum at $(\mathbf{x}, t)$ before collision. $m^{\sigma}$ is the molecular mass of the $\sigma$ th component.

In order to simulate multiple component fluids or fluids with nonideal gas equations of state, nonlocal interactions among the particles must be incorporated. Hence $\mathbf{u}^{\sigma}$ will be determined differently from the prescription above. In this new model, we present an efficient way for introducing this kind of nonlocal interaction. We define an interaction potential,

$$
V\left(\mathbf{x}, \mathbf{x}^{\prime}\right)=G_{\sigma \bar{\sigma}}\left(\mathbf{x}, \mathbf{x}^{\prime}\right) \psi^{\sigma}(\mathbf{x}) \psi^{\bar{\sigma}}\left(\mathbf{x}^{\prime}\right),
$$

where $G_{\sigma \bar{\sigma}}\left(\mathbf{x}, \mathbf{x}^{\prime}\right)$ is a Green's function. The quantity $\psi^{\sigma}$ $\left[=F^{\sigma}\left(n^{\sigma}\right)\right]$ plays a role as the effective number density for component $\sigma$ with its physical significance seen clearly below. For the purpose of dealing with the problems concerned here, it is sufficient to only involve nearestneighbor interactions, namely

$$
G_{\sigma \bar{\sigma}}\left(\mathbf{x}-\mathbf{x}^{\prime}\right)= \begin{cases}0, & \left|\mathbf{x}-\mathbf{x}^{\prime}\right|>c \\ \mathcal{G}_{\sigma \bar{\sigma}}, & \left|\mathbf{x}-\mathbf{x}^{\prime}\right|=c .\end{cases}
$$

The magnitude of $\mathcal{G}_{\sigma \bar{\sigma}}$ controls the strength of the interaction potential between components $\sigma$ and $\bar{\sigma}$, while its sign determines whether it is attractive or repulsive.

Having the interaction potential defined, the rate of net momentum change induced at each site is simply

$$
\frac{d p^{\sigma}}{d t}(\mathbf{x})=-\psi^{\sigma}(\mathbf{x}) \sum_{\bar{\sigma}=1}^{S} \mathcal{G}_{\sigma \bar{\sigma}} \sum_{a=0}^{b} \psi^{\bar{\sigma}}\left(\mathbf{x}+\hat{\mathbf{e}}_{a}\right) \hat{\mathbf{e}}_{a}
$$

which equals $-\nabla V$ as $c \rightarrow 0$. Therefore, after a collision, the new net momentum at site $\mathbf{x}$ for the $\sigma$ th component now becomes

$$
\rho^{\sigma}(\mathbf{x}) \mathbf{u}^{\sigma}(\mathbf{x})=\rho^{\sigma}(\mathbf{x}) \mathbf{u}(\mathbf{x})+\tau_{\sigma} \frac{d p^{\sigma}}{d t}(\mathbf{x}),
$$

where $\rho^{\sigma}(\mathbf{x})=m^{\sigma} n^{\sigma}(\mathbf{x})$ is the mass density of the $\sigma$ th component. Apparently, with the interaction potential, the collision does not conserve the net momentum at each site. This may be the most distinct feature of this model. However, it can be shown directly that the total momentum of the system obtained by summing over the net momenta at every site is still exactly conserved, provided $\mathcal{G}_{\sigma \bar{\sigma}}$ is a symmetric $S \times S$ matrix, and no net momentum exchange has occurred at the boundary. Therefore, the momentum conservation is achieved in a more general sense than the local sitewise momentum conservation.

We demonstrate below that this dynamical rule exhibits thermodynamic phase transitions for a single component $(S=1)$ case. Multiplying Eq. (1) by 1 and $\hat{\mathbf{e}}_{a}$ and summing over $a$, after substituting the functional form from Eq. (3) for $n_{a}^{\sigma(e q)}$ and some straightforward algebra, we obtain

$\frac{\partial \rho}{\partial t}+\nabla \cdot(\rho \mathbf{u})=0$

$\rho\left(\frac{\partial}{\partial t}+\mathbf{u} \cdot \boldsymbol{\nabla}\right) \mathbf{u}+\frac{\left(1-d_{0}\right) c^{2}}{D} \nabla \rho-\nabla \cdot[\mu \boldsymbol{\nabla} \mathbf{u}]$

$$
+\nabla[\zeta \nabla \cdot \mathbf{u}]=-\nabla V,
$$

at low frequency and long-wavelength limit. Without $-\nabla V$ on the right-hand side of the second equation, the momentum equation above is the usual Navier-Stokes equation with an isothermal ideal gas equation of state,

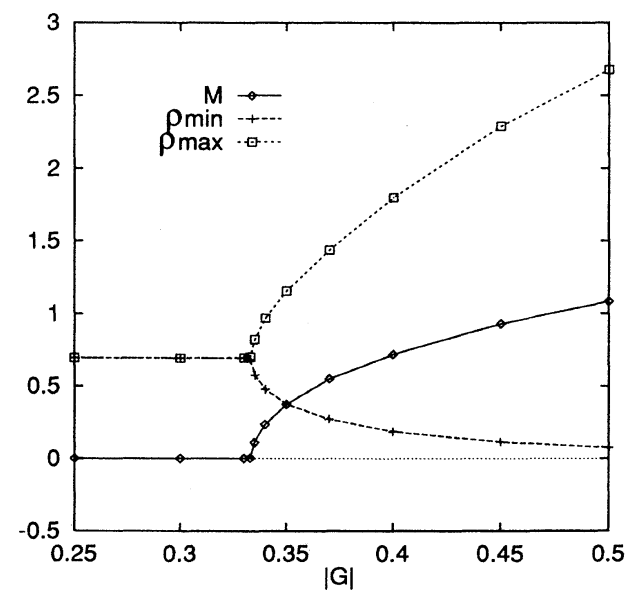

FIG. 1. Maximum and minimum density values $\left(\rho_{\max }\right.$ and $\left.\rho_{\min }\right)$, and the order parameter $\mathcal{M}$ as functions of $\mathcal{G}$. Phase separation, indicated by the bifurcation of the system from homogeneous into two-phase, occurs at the analytically calculated critical value $\mathcal{G}=-1 / 3$. 
and viscosities $\mu=\mu(\rho)$ and $\zeta=\zeta(\rho)[3,4]$. The explicit form of the additional term can be derived from Eq. (7) in the long-wavelength limit as

$$
V=\frac{b c^{2}}{2 D} \mathcal{G} \psi^{2}
$$

where $\mathcal{G}=\mathcal{G}_{11}$ for the single-component fluid. Combining the two gradient terms in Eq. (9), the equation of state is ready to be written down as

$$
p=\frac{c^{2}}{D}\left[\left(1-d_{0}\right) \rho+\frac{b}{2} \mathcal{G} \psi^{2}(\rho)\right],
$$

which is, in general, not an ideal gas equation of state. The second term on the right-hand side adds an effective potential tail to the elastic interaction represented by the first term. Consequently, thermodynamic phase

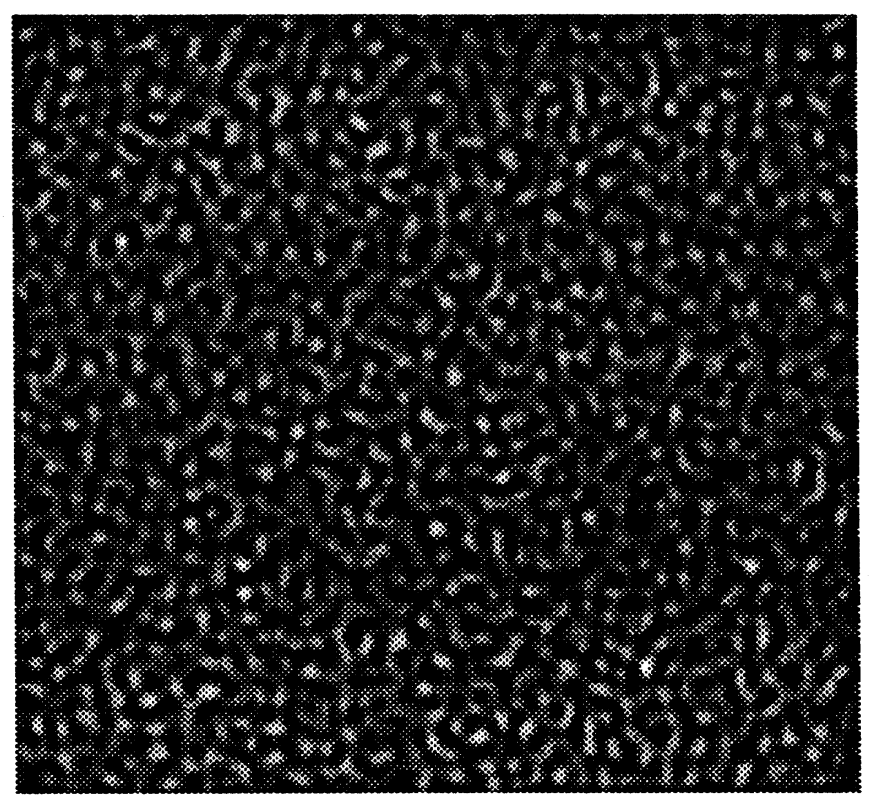

(a)

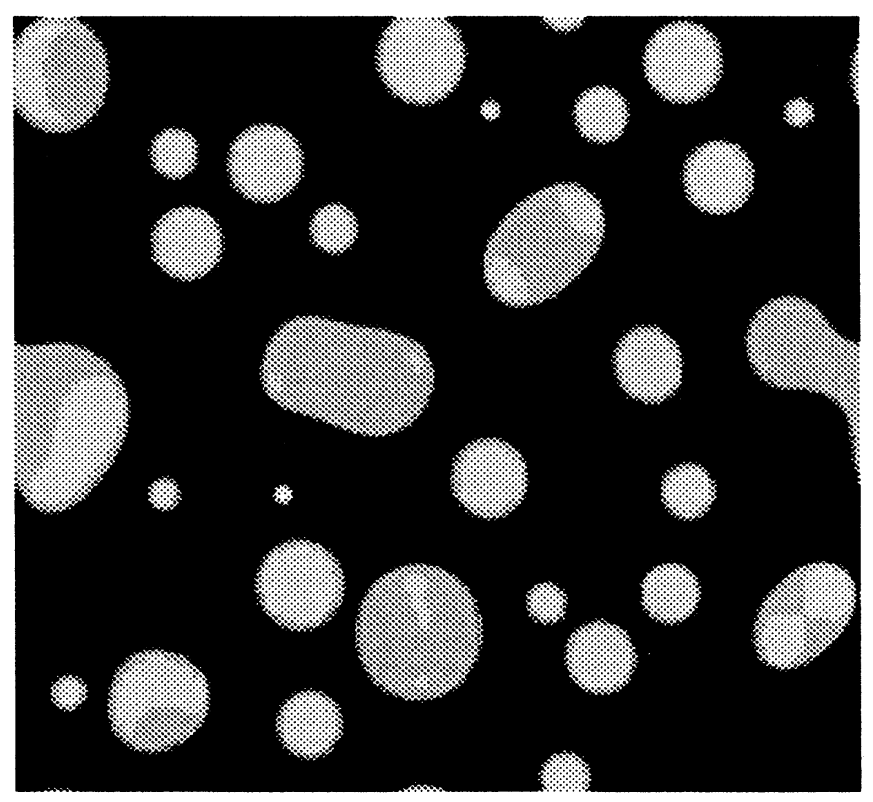

(c)

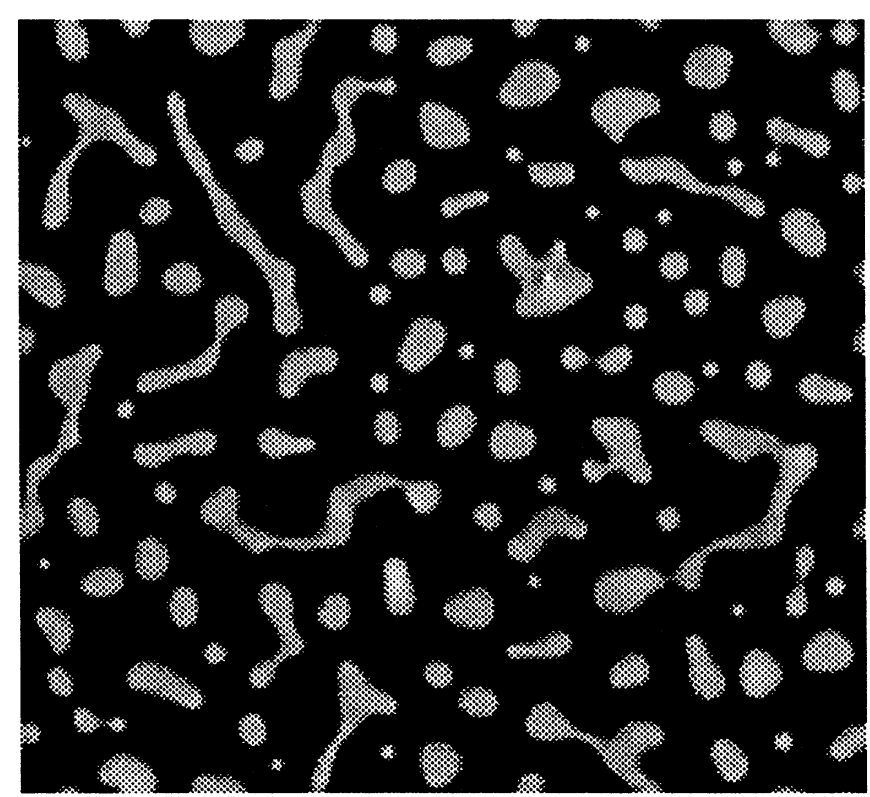

(b)

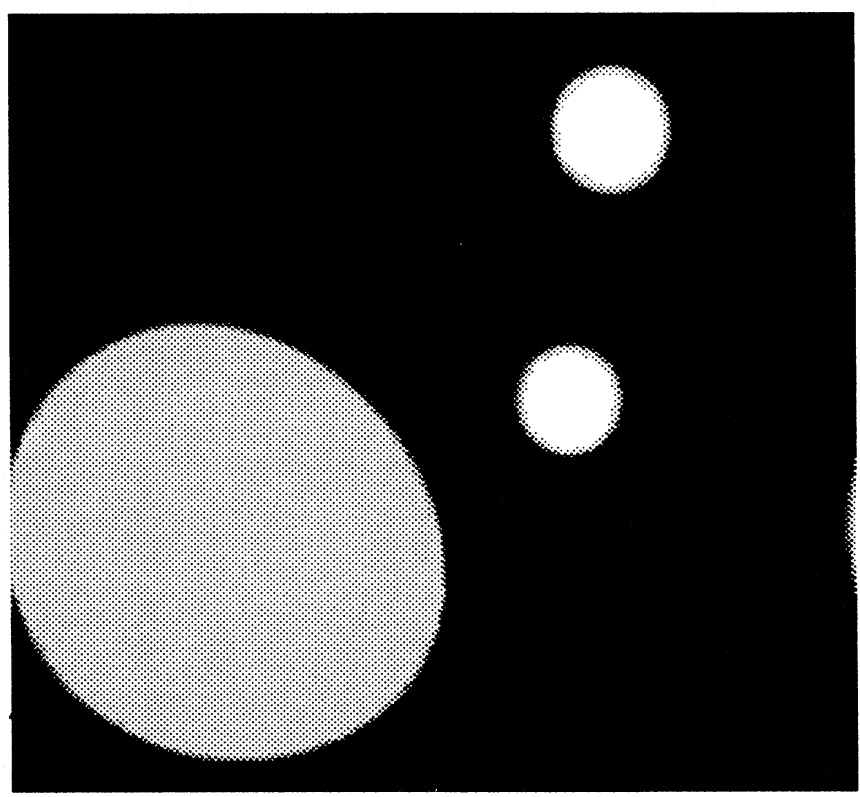

(d)

FIG. 2. Phase transition in a LB simulation on a $256 \times 256$ lattice with a single component. Shown are the time evolution of the number density distribution at $t=20$ (a), $t=200$ (b), $t=2000$ (c), and $t=20000$ (d). The variation of the density is shown in gray scale with the minimum in black and the maximum in white. Since the lattice is hexagonal, the graphics are distorted by a factor of $\sqrt{3} / 2$ in one direction. $\mathcal{G}=-0.45, \rho_{0}=1$, and $\langle\rho\rangle=0.693 \cong \ln 2$. 
transitions will occur if $p$ is not a monotonically increasing function of the density $\rho$. This will be possible, according to the above expression, if $\mathcal{G}$ (which is related to temperature) is sufficiently negative and $\psi$ is a monotonically increasing but bounded function of $\rho$; therefore, $V$ is bounded too.

We now present some results of numerical simulations for a two-dimensional system in which a first-order phase transition occurs. For simplicity, we have chosen $\psi=$ $\rho_{0}\left[1-\exp \left(-\rho / \rho_{0}\right)\right]$, where $\rho_{0}$ is a constant. The critical value can be obtained analytically as $\mathcal{G}_{c}=-4\left(1-d_{0}\right) / b \rho_{0}$ at $\rho_{c}=\rho_{0} \ln 2$. Below this critical value, there will exist a range of $\rho$ values in which $d p / d \rho$ is negative, which signals the occurrence of a phase transition. The numerical simulations reported here are on a $2 \mathrm{D} 256 \times 256$ hexagonal lattice; and we took $d_{0}=0.5, \rho_{0}=1.0$ and $\langle\rho\rangle=\rho_{0} \ln 2 \cong 0.693$. Hence the critical value is at $\mathcal{G}_{c}=-1 / 3$. ( \langle\rangle is a spatial average over the entire lattice.) Initially the density distribution is homogeneous and with a small (1\%) random perturbation. Figure 1 is a plot of the maximum and minimum densities, and the order-parameter $\mathcal{M} \equiv \sqrt{\left\langle(\rho-\langle\rho\rangle)^{2}\right\rangle} /\langle\rho\rangle$ in the final steady state as functions of the parameter $\mathcal{G}$. As $\mathcal{G}$ gets below the critical value, the system changes from a single-phase fluid to a two-phase fluid. The numerically measured bifurcation point is between $\mathcal{G}=-0.333$ and -0.335 . Displayed in Fig. 2 in gray scale are the evolution of the mass density distributions at $\mathcal{G}=-0.45$. To be seen is the separation of two different thermodynamic phases and the existence of surface tension. This calculation takes $0.012 \mathrm{~s}$ for each step on a $16 \mathrm{~K}$ processor CM-200 machine.

Besides having the capability of modeling multiphase flows, this model can also simulate fluids with immiscible components. For demonstrating this effect, here we consider briefly a system of $S$ mutually equal repulsive components. That is, $\mathcal{G}_{\sigma \sigma}=0$ and $\mathcal{G}_{\sigma \bar{\sigma}}=\mathcal{G}$ for $\sigma \neq \bar{\sigma}$; and $\psi^{\sigma}=n^{\sigma}$. Clearly for this case, the Navier-Stokes equations are satisfied with an ideal gas equation of state in each single-component region [8]. The interesting dynamics only occur when different components coexist in the same region. For the sake of analytical simplicity, if we also choose $\tau_{\sigma}=\tau$ and $m^{\sigma}=m$, then an equation governing the evolution of the infinitesimal perturbation of a spin field [17] can be obtained:

$$
\frac{\partial(n A)}{\partial t}+\boldsymbol{\nabla} \cdot(\mathbf{u} n A)=\boldsymbol{\nabla} \cdot[\eta \nabla(n A)],
$$

where the spin field, $A$, is defined as

$$
n A \equiv \sum_{a} \sum_{\sigma} \sigma n_{a}^{\sigma}
$$

In this case, the spin diffusivity, $\eta$, can be shown to have the following form:

$$
\eta=\frac{c^{2}}{D}\left[\left(\tau-\frac{1}{2}\right)-\frac{\tau b}{m S} \mathcal{G} n\right] .
$$

Therefore, when $\mathcal{G}$ is large enough $[\geq(\tau-1 / 2) m S / \tau b n]$ the diffusivity will become negative. This indicates a separation of components, which is the basic property of immiscible fluids [8]. Numerically we have indeed observed this phenomenon on a $2 \mathrm{D} 256 \times 256$ hexagonal lattice for the two-component case. We have chosen $m=1, \tau=1$, $\langle n\rangle=1$, and $\left\langle n^{1}\right\rangle /\left\langle n^{2}\right\rangle=4 / 6$. Therefore, $\mathcal{G}_{c}=1 / 6$. The time evolution of the component patterns is qualitatively similar to that in Fig. 2 for the density distribution for the two-phase one-component case. Figure 3 is the surface plot of the total number density distribution $n$, of this two-component system at time $t=20000$ with $\mathcal{G}=0.25$. Component 1 is seen to have separated from component 2 and formed circular "drops." Figure 4 is a measure, from Fig. 3, of the total density difference inside and outside a single-component drop, as a function of the inverse drop radius. Since the pressure for this case is proportional to the total density inside and outside a drop, it is seen that

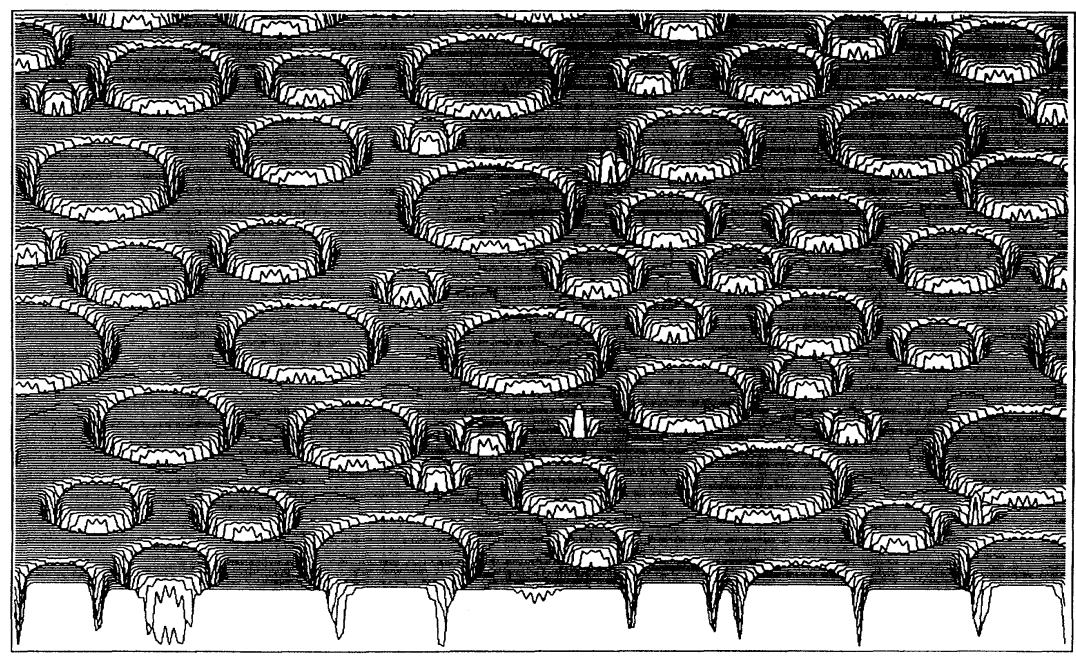

FIG. 3. Total density distribution of the two-component system on a $256 \times 256$ lattice at time $t=20000$ and $\mathcal{G}=0.25$. 


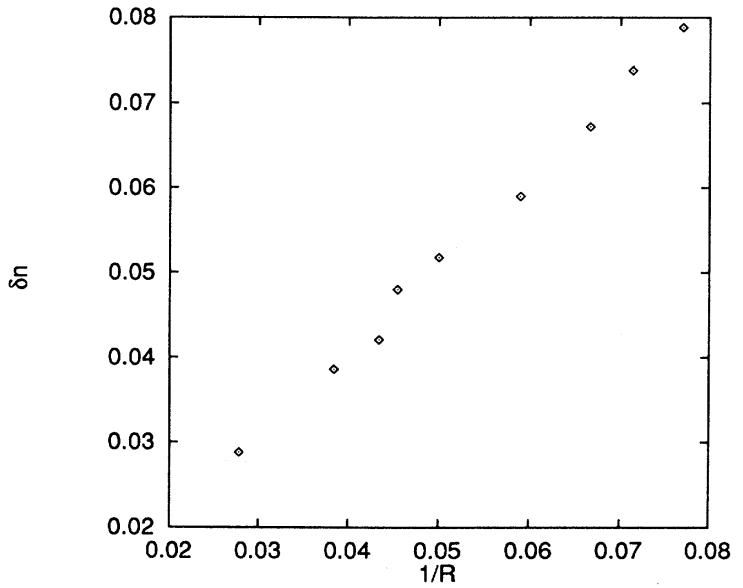

FIG. 4. The density difference inside and outside a drop as a function of the inverse drop radius for the two-component system at $t=20000$ and $\mathcal{G}=0.25$.

Laplace's law $[8,11]$,

$$
p_{\text {in }}-p_{\text {out }}=\frac{\lambda}{R}
$$

is satisfied within the accuracy of our measurement, where $p_{\text {in }}$ and $p_{\text {out }}$ are, respectively, the pressures inside and outside a single-component drop of radius $R$.

In this paper, we have presented a LB model which has the capability of simulating multiphase and multicomponent immiscible fluids with different masses in constant temperature. The fundamental feature in this model is the introduction of an interparticle potential, which effectively adds an attractive or repulsive tail to the sitewise elastic collision. By selecting the appropriate values of the matrix $\mathcal{G}_{\sigma \bar{\sigma}}$ and $\psi^{\sigma}$, the properties of this potential can be varied so that the various fluids can be simulated. The detailed description and study of this model will be presented in a future paper [18]. One of the major improvements to this model would be to extend it to include a dynamical temperature. Due to perhaps the discretized nature of the "lattice Boltzmann" approach, there are some small anomalous velocities observed, as reported in other multi-phase models [19], in the interfacial region which is subjected to further studies. Because of the fact that the LB method is naturally suitable for massively parallel computation, this model can be used for large-scale simulations of complex fluids in various specific cases and boundary conditions. We have also found, for the single-component case, flows with Reynolds number up to $10^{4}$ can be simulated. With all these unique features, many potential scientific and industrial applications of this model can be expected.

The authors wish to thank Dr. Gary Doolen, Dr. David Montgomery, Dr. Lishi Luo, Dr. Yue Hong Qian, Dr. Francis Alexander, Dr. Shiyi Chen, and Dr. Graham Wallis for their valuable discussions and suggestions. We also thank the Advanced Computing Laboratory at Los Alamos National Laboratory for providing us with the privilege for using the CM-200 computer. This work was partially supported by the U.S. Department of Energy through Los Alamos National Laboratory, and at Dartmouth by U.S. Department of Energy Grants No. DEFG02-85ER53298 and No. DE-FG02-85ER53194 and by NASA Grant No. NAG-W-710.
[1] G. McNamara and G. Zanetti, Phys. Rev. Lett. 61, 2332 (1988).

[2] F. J. Higuera and J. Jimenez, Europhys. Lett. 9, 663 (1989); S. Succi, R. Benzi, and F. Higuera, Physica D 47, 219 (1991).

[3] H. Chen, S. Chen, and W. H. Matthaeus, Phys. Rev. A 45, 5339 (1992).

[4] Y. H. Qian, D. d'Humières, and P. Lallemand, Europhys. Lett. 17, 479 (1992).

[5] U. Frisch, B. Hasslacher, and Y. Pomeau, Phys. Rev. Lett. 56, 1505 (1986).

[6] U. Frisch, D. d'Humières, B. Hasslacher, P. Lallemand, Y. Pomeau, and J.-P. Rivet, Complex Systems 1, 649 (1987).

[7] S. Wolfram, J. Stat. Phys. 45, 471 (1986).

[8] D. H. Rothman and J. Keller, J. Stat. Phys. 52, 1119 (1988).

[9] H. Chen, S. Chen, G. Doolen, Y. C. Lee, and H. Rose,
Phys. Rev. A 40, 2850 (1989).

[10] C. Appert and S. Zaleski, Phys. Rev. Lett. 64, 1 (1990).

[11] A. K. Gunstensen, D. H. Rothman, S. Zaleski, and G. Zanetti, Phys. Rev. A 43, 4320 (1991).

[12] S. Chen, G. Doolen, K. Eggert, D. Grunau, and E. Y. Loh, Phys. Rev. A 43, 245 (1991).

[13] S. Chen, H. Chen, D. Martinez, and W. H. Matthaeus, Phys. Rev. Lett. 67, 3776 (1991).

[14] P. L. Bhatnagar, E. P. Gross, and M. Krook, Phys. Rev. 94, 511 (1954).

[15] Y. H. Qian, Ph.D. thesis, Université de Paris, 1990.

[16] F. Alexander, H. Chen, and S. Chen, Phys. Rev. A 46, 1967 (1992).

[17] H. Chen and W. H. Matthaeus, Phys. Fluids 30, 1235 (1987).

[18] X. Shan and H. Chen (unpublished).

[19] A. Gunstensen, Ph. D. thesis, MIT, 1992. 


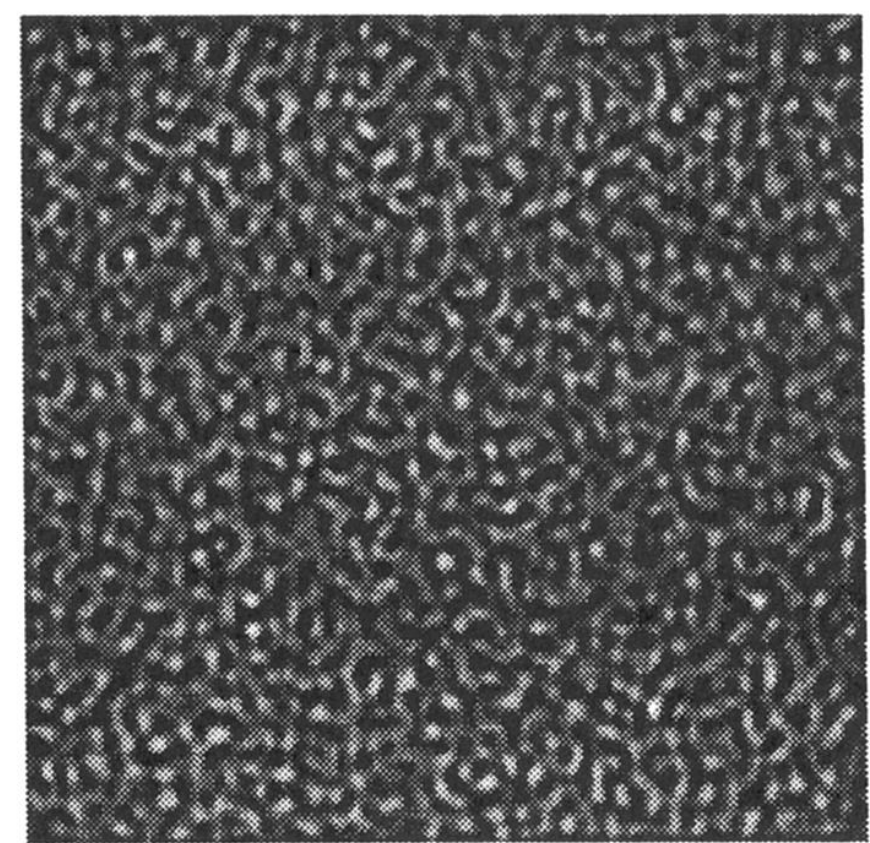

(a)

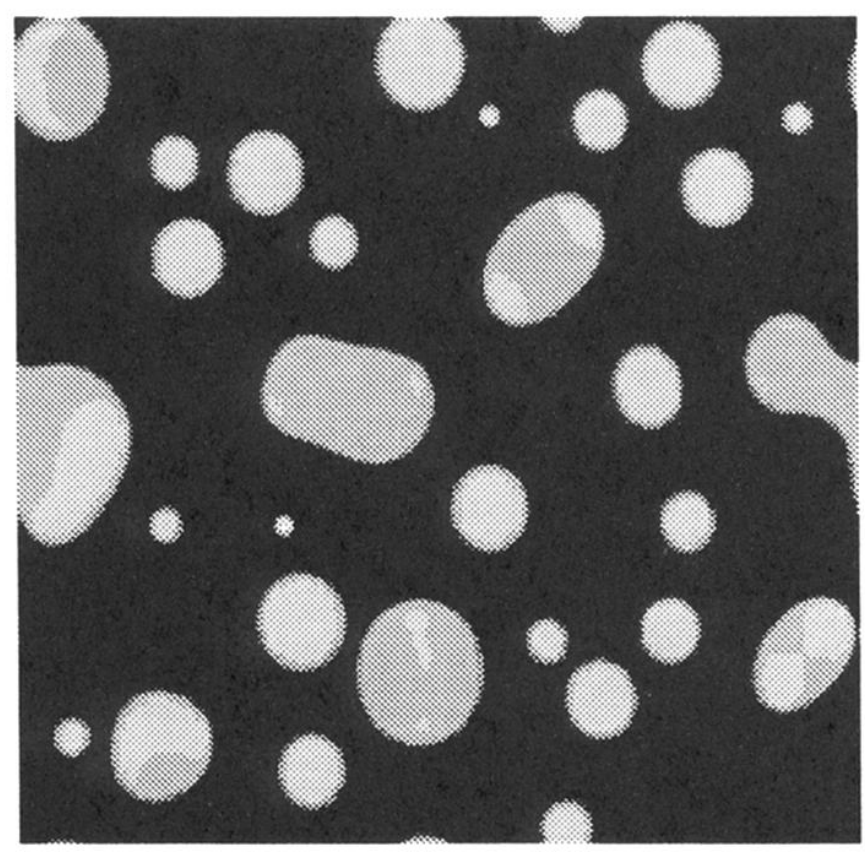

(c)

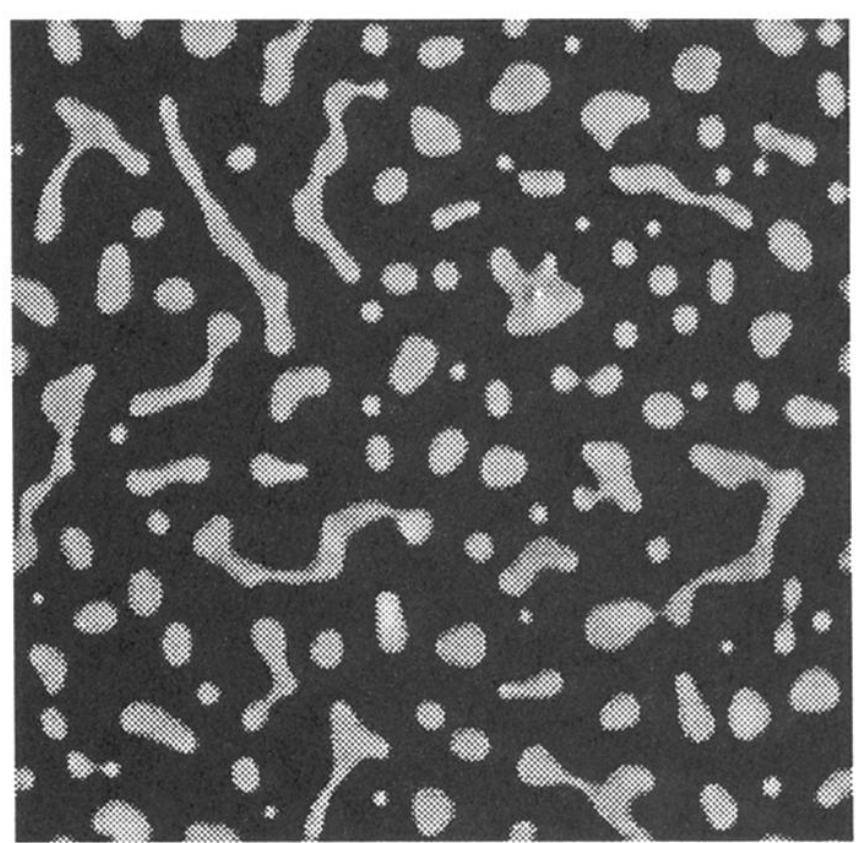

(b)

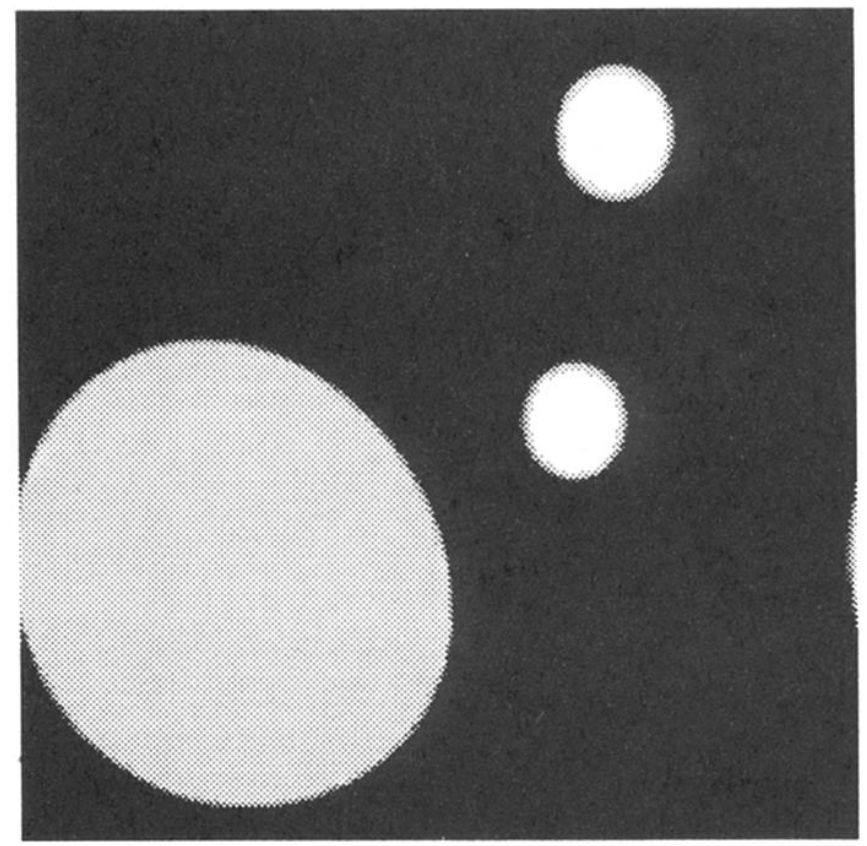

(d)

FIG. 2. Phase transition in a LB simulation on a $256 \times 256$ lattice with a single component. Shown are the time evolution of the number density distribution at $t=20$ (a), $t=200$ (b), $t=2000$ (c), and $t=20000$ (d). The variation of the density is shown in gray scale with the minimum in black and the maximum in white. Since the lattice is hexagonal, the graphics are distorted by a factor of $\sqrt{3} / 2$ in one direction. $\mathcal{G}=-0.45, \rho_{0}=1$, and $\langle\rho\rangle=0.693 \cong \ln 2$. 